\title{
A Dynamic Context-Aware Access Network Selection for Handover in Heterogeneous Network Environments
}

\author{
Peyman TalebiFard and Victor C.M Leung \\ Department of Electrical and Computer Engineering \\ The University of British Columbia \\ Vancouver, BC \\ Email: $\{$ Peymant,VLeung $\} @$ ece.ubc.ca
}

\begin{abstract}
Context-awareness is a key ingredient in any ubiquitous and pervasive system and provides intelligence to the system, allowing computing devices to make appropriate and timely decisions on behalf of users. One of the important aspects of mobility management is the dynamic selection of the best access network for a multimodal device when there is a need to perform a handover. Multi Attribute Decision Making (MADM) is one of the successfully used methods in the literature to solve decision making problems. The problem of access network selection has been addressed by decision making methods based on available network information. However, the quality of information is not considered. Weighted Product Method (WPM) is an MADM method that penalizes the unreliable attributes in making a decision. It does not suffer from ranking abnormalities and its cheaper computational cost makes it a suitable candidate for decision making in a dynamic situation. In this paper, an algorithm for a context-aware network selection is proposed that is based on a modified WPM for access network selection. We use a weight distribution method based on sensitivity analysis of WPM for the most influential criteria based on the state of user at a given time. Our evaluation is based on comparing WPM with TOPSIS that is successfully used in many decision making problems.
\end{abstract}

Keywords: Access network selection, Context awareness, Handover, MADM, pervasive computing, Weighted Product Method.

\section{INTRODUCTION}

As mobile communication networks become more prevalent, and advances in wireless devices enable multiple wireless technologies to be supported, the issue of context-aware mobility management becomes a challenge. Context-aware mobility is the ability to dynamically capture and use the surrounding contextual information of a mobile entity to improve the performance of the system. Context-awareness is therefore a key ingredient in any ubiquitous and pervasive system and provides intelligence to the system, allowing computing devices to make appropriate and timely decisions on behalf of users. Contextual information is utilized in many areas such as e-Healthcare systems [1]. One of the important aspects of mobility management is the dynamic selection of the best access network for a multimodal device when there is a need to perform a handover.
The Internet Engineering Task Force (IETF) in mobility related terminology [2] refers to context as the information required to transfer the current state of a routing-related service on re-establishing a new connection to a subnet for a similar service without having to perform the entire protocol exchange with the device. However, new technologies have enabled more information to be accessible with various context information providers and sensors. Beyond this, context awareness for mobility management purposes can be thought of as services that a user often uses, features and requirements that those services demand, security requirements of services and of the user, capabilities of the device, network related information, mobility information such as speed, and coverage of available access networks.

Existing works in the literature deal with the problem of Access Network Selection (ANS) with Multi Attribute Decision Making (MADM) methods such as Technique for Order Preference by Similarity to Ideal Solution (TOPSIS). The scope of previous work is mainly on the network selection prior to the connection establishment of the user terminal. One of the advantages of TOPSIS is its accuracy and contrast in ranking the best alternative. However, for making decision in a dynamic situation for the purpose of selecting the appropriate access network in a dynamic situation, it is computationally expensive and suffers from ranking abnormality as the alternatives are being removed or added. The ranking method in TOPSIS is dependent on the other alternatives since this method is based on the shortest distance from the best alternative and longest distance from the worst alternative. Weighted Product Method (WPM) is a more rigorous method in penalizing the alternatives with least significance, and computationally cheaper than TOPSIS method. It is dimensionless and ranking abnormality issue does not apply to WPM. The preference index of each alternative is independent of the other alternatives and one can set a threshold for an acceptable preference index to minimize the number of unnecessary handovers. Therefore, we recommend WPM as a better alternative than TOPSIS for dynamic decision making situations. Furthermore, the decision making methods that are proposed in the literature take into account the 
available network information and attributes. Beyond using the network information, other contextual information will be available by mobile entities and other sensors. Therefore, in a context-aware approach the system is dealing with a large amount of information. Taking into account the quality of collected information is the key in developing a more effective context management platform.

In this paper, we address the problem of dynamic contextaware ANS. We take into account the Quality of Context (QoC) and propose a dynamic context-aware solution to the ANS problem that is based on a modified WPM. In a dynamic situation, for instance, when a handover decision is to be made, it is important to choose the appropriate access network to continue the session with the existing context of the mobile user. In this method, the QoC is utilized to penalize alternatives that have poor quality data. The proposed weight distribution method not only depends on the QoC parameters, but also on the fuzzy measure of the saliency of the context information that implies a truth value measure for a set of measurements for a predicate.

In this paper we assume that attributes belong to independent axis of measurements. In evaluating the decision making methods, there are two important evaluative criteria according to Triantaphyllou in [3]. The first one is that any accurate decision method for a multi-dimensional problem should also be accurate when applied to a single dimension problem and the second criterion is that the rank of the best alternative should not change when a worse alternative is replaced by another worse alternative, the so called ranking abnormality.

This paper is organized as follows. Section II describes the system architecture that our proposed method is based on. In Section III we provide the modelling and notations that will be used in the paper. In Section IV we present our method that is based on WPM and fuzzy measure of the saliency of the context information. Section V provides the evaluation and demonstration of the proposed solution. Finally, Section VI concludes the paper.

\section{Proposed Context-Aware System Architecture}

For the purpose of this paper we simplify the representation of a context-aware service platform to a typical Service Oriented Architecture (SOA) [4]. Such a platform can be divided into three major parts. Service registry and management, Service consumer, and Service provider. Service registry and management has the role of context processing, aggregation, classification and inter-domain context handling. Service consumers are mobile devices and laptops that operate based on the provided services. Service providers can have the role of context information providers and service enablers for Location Based Services (LBS), charging, and other contextaware applications.

Based on this framework, we assume that services, and applications can also initiate handover for the case of Machine to Machine (M2M) interaction. In order to ensure the availability of contextual information that can be utilized to support the operation of heterogeneous context-aware services, the following context management capabilities are needed:

- Context sensing and collection: consists of collecting the required contextual information from different context sources such as device, network, and other sensors.

- Context processing: is the inference of a situation from raw data that are collected from different entities. It requires reasoning and inference methods to infer higher level information from lower level contextual information.

- Inter-domain context handling and aggregation: is the process of managing and aggregating the context information collected from various sources and representing that in an easy to use and understandable way to be shared with other entities.

- Context distribution: is the process of disseminating and publishing the context information to applications and services based on the QoC agreement level.

- QoC mapping function: is in charge of managing and provisioning of the QoC parameters to ensure the required Quality of Service (QoS) and Quality of Experience (QoE) expected.

\section{A. QoC agreement}

In a service oriented approach, there are several actors within each role of service registry and management, service consumer and service provider. Analogous to the Service Level Agreement (SLA) that is an agreement to provide a certain QoS, the QoC should also be negotiated among the entities. Such an agreement can be between the service providers and service consumers or users. It is also possible that a context service provider subscribe to another context provider on behalf of others. In these cases, the contextaware service providers need to ensure that the quality of obtained information is beyond a certain threshold. QoC can furthermore be a criterion for selection of appropriate context providers.

\section{Modeling And Defining Context}

Various definitions of context have been given in the literature[5][6][7]. Context is defined as user related information that is used to describe the state of a user or system in a specific situation.

For simplicity of notation we use the following notation to indicate the context state of the user at any given time $t$ as an $\mathrm{N}$-dimensional vector:

$$
C_{i}^{t}=\left(a_{1}^{t}, a_{2}^{t}, \ldots, a_{N}^{t}\right)
$$

where context attribute $a_{i}^{t}$ is the $i^{t h}$ attribute of a context state at time $t$.

Context space $R_{i}=\left(a_{1}^{R}, a_{2}^{R}, \ldots a_{N}^{R}\right)$ is the domain of acceptable values that are allowed for a specific context attribute. An acceptable region $a_{i}^{R}$ is defined as a set of elements $V$ that satisfies a predicate $P$ such that $a_{i}^{R}=\{V \mid P(V)\}$ [5][6]. For the scope of this paper the context space is a set of predefined regions of acceptable operating modes or states that a user 
terminal or application can be in that state. It is the range of acceptable values that are allowed for a specific context attribute. For the purpose of this work, we define regions as predefined modes (profiles) such as low bandwidth profile, low cost profile, secure profile and etc.

It is also very important to develop a data format that can be used across different domains for communicating the context of users. One possible format is the Presence Information Data Format (PIDF) that is standardized by IETF in RFC 3863 [8]. The PIDF data format is not tied to any protocol for transporting it. In IETF and IP Multimedia Subsystem (IMS), PIDF can typically be transported using the Session Initiation Protocol (SIP) [9], or other protocols such as the Hypertext Transfer Protocol (HTTP) or Simple Object Access Protocol (SOAP) [10].

\section{A. Quality of Context}

In developing context-aware services and provisioning of services, the availability and reliability of contextual information is of great importance. The QoC is any information related to the quality of contextual information that are involved in making context-aware decisions [11][12][13]. Since context information can often be uncertain and incomplete in nature, it is important to provision the enforced actions based on the QoC to ensure the effective utilization of provided context information that leads to efficient context management solutions. Authors in [11] and [12] have proposed a quantification approach of QoC. Furthermore, an algorithm for evaluation of QoC is also presented in [12] and the following parameters of QoC are evaluated.

1) Precision: refers to the level of accuracy. For example, a GPS receiver can locate a user with the precision of less than 10 meters, while positioning a user via a GSM cellular network may have a precision of up to 500 meters [13]. We denote the precision of collected information about attribute $a_{i}$ as $P\left(a_{i}\right)$.

2) Probability of correctness: refers to the probability of correctness for any given contextual information. For the previously mentioned example, there is no guarantee that the precision is true since it may depend on various other factors such as the density of the base stations in a specific area. Let $\operatorname{PrC}\left(a_{i}\right)$ denote the probability of correctness about attribute $a_{i}$. An example of this can be collected information about the weather condition in a city. If the collected information is originated from a mobile device, it may not be correct since the mobile device can be located indoor at that time.

3) Completeness: is a representation of the degree of support that a set of attributes provide for inferring a context. Let $C\left(a_{i}\right)$ denote the completeness of attribute $i$, then it can be represented by the proportion of the weights of all features that support a predicate with respect to all the features.

4) Trust-worthiness: is an indication of the likeliness that the provided information is correct. It is analogous to the notion of rating in the context of sellers and customers.
Let $T\left(a_{i}\right)$ denote the trustworthiness of the $i^{t h}$ attribute and it can be measured in terms of the accuracy of the information, the previous history of collected data and statistical estimation techniques.

5) Resolution: refers to the granularity of the provided information and can be denoted by $R\left(a_{i}\right)$.

6) Up to datedness and time validity of information: refers to the age of the collected and provided information. For many applications, the events are time stamped and the age of the provided data play a major role. Denoting $U\left(a_{i}\right)$ as the time validity of a context information, it is represented in terms of the difference of the current time and most recent measurement time.

QoC can be communicated among the network entities either as metadata or separately. Our assumption in this paper is based on metadata method of communication where the QoC for each sensed data is transmitted with the data.

For the purpose of this paper we define a measure of saliency for a context information. It is an indication of the containment of attributes for inferring a predicate and the truth value of that predicate is based on the QoC parameters. The truth value function for a set of attributes on a predicate returns a truth value $\in[0,1]$.

$$
\mu: R^{m} \rightarrow[0,1]
$$

for $m$ dimensions such as precision, trust-worthiness, completeness, timeliness, etc. The truth value of a predicate or a context information $a$ is $\mu(a)$ and it is a function of the aforementioned QoC parameters. i.e.

$$
\mu_{i}(a)=F\left(P\left(a_{i}\right), \operatorname{Pr} C\left(a_{i}\right), C\left(a_{i}\right), T\left(a_{i}\right), R\left(a_{i}\right), U\left(a_{i}\right)\right)
$$

where $i=1,2, \ldots N$ and $\mu_{i}(a)$ represents the truth value of context attribute $a$ collected from source $i$.

\section{B. The Problem of Context-aware Access Network Selection}

We intend to develop a utility based data fusion method for the purpose of access network selection in a heterogeneous environment based on a MADM approach. For the purpose of developing the appropriate data fusion model, we need to develop our model of context. Some of the important issues in modeling the context information are relevance of each attribute, data structure and representation of data, quality of collected information, and cost of capturing the data.

In inferring a situation or state, essential attributes are those that may have a negative influence if missing or their value is not within the acceptable region of a predefined situation and optional attributes are the attributes that are complementary in inferring a situation.

\section{Weighted Product Method}

WPM [14] is a compensatory MADM technique that penalizes the alternatives that have unreliable or poor attribute values by assigning appropriate weights. Each row $A_{i}$ in the decision matrix $D M$ corresponds to an access network alternative. A typical procedure for WPM is as follows: 
1) Determine the weight of each attribute for a given context vector and normalize weights such that

$$
\sum_{j=1}^{n} w_{j}=1
$$

where $w_{j}$ is the weight of $j^{\text {th }}$ attribute.

2) For each alternative $A_{i}$ in the decision matrix $D M$, raise each element to the power equal to the weight of each attribute.

$$
D M=\left[\begin{array}{ccccc}
\left(a_{11}\right)^{w_{1}} & \ldots & \left(a_{1 j}\right)^{w_{j}} & \ldots & \left(a_{1 n}\right)^{w_{n}} \\
\left(a_{21}\right)^{w_{1}} & \ldots & \left(a_{2 j}\right)^{w_{j}} & \ldots & \left(a_{2 n}\right)^{w_{n}} \\
\ldots & \ldots & \ldots & \ldots & \ldots \\
\left(a_{m 1}\right)^{w_{1}} & \ldots & \left(a_{m j}\right)^{w_{j}} & \ldots & \left(a_{m n}\right)^{w_{n}}
\end{array}\right]
$$

3) The best alternative is the row with the highest product of elements:

$$
D M^{*}=\left\{A_{i} \mid \max _{i}\left(\prod_{j=1}^{n}\left(a_{i j}\right)^{w_{j}}\right\}\right.
$$

\section{A. Determining the Weights of Attributes in Making Decisions}

Deploying any compensatory MADM requires an appropriate weight assignment mechanism. The proposed mechanism in this paper is based on the types of attributes as discussed above and the QoC parameters. For the scope of this work, weights of attributes are relevant from two perspectives:

1) Having known the state of a user at any given time $t$, we want to rank the access network alternatives and make the optimal ANS.

2) Having collected the contextual information from various sources, we need to infer the state of the user.

The state of the user is chosen among a set of predefined modes or profiles.

\section{B. Weight Assignment Based on The Most Critical Attribute}

As mentioned in the previous sections, the context information vector can represent either the state of the user or the context of the candidate access network alternatives. In the decision making approach proposed in this paper a context-aware choice of access network based on the context information vector of the user and the aggregated context of access network and available services is made. In making such decisions, there are attributes that are critical in making decisions based on the current status (profile) of the user and the application or services that the user is intending to access. On the other hand, it is important to eliminate the unnecessary changes in access network (handover) to reduce the cost of unnecessary signalling that can lead to degradation of QoE perceived by the user in many use case scenarios such as multimedia streaming, or voice/video calls. This is to ensure that the most critical attribute has the most significant effect in ranking of the alternatives. In our proposed algorithm we deploy a weight distribution method that is based on the degree of criticality of attributes relevant to a specific situation (profile).
Weight assignment is based on a sensitivity analysis of the aforementioned MADM algorithm on different attributes. By utilizing this weight distribution approach we guarantee that the most critical attribute has the greatest influence in ranking alternatives.

\section{Weighted Product Method with Interval Data}

Since the collected contextual information are fuzzy in nature, one possible approach is to show them in the form of interval data. The acceptable region or possible modes of operation can also be characterized with interval data. Attributes and characteristics of each mode can have lowest acceptable value, or a range of acceptable values or if it is cost or delay, the highest acceptable value would be applicable. For convenience of notation, we represent the attributes of each operation mode as an interval of lowest to highest possible values. The following matrix $\widetilde{D M}$ shows the decision making matrix in the form of interval data where columns are the attributes and rows are the access network alternatives:

$$
\widetilde{D M}=\left[\begin{array}{ccccc}
\left(\underline{a}_{11}, \bar{a}_{11}\right) & \ldots & \left(\underline{a}_{1 j}, \bar{a}_{1 j}\right) & \ldots & \left(\underline{a}_{1 n}, \bar{a}_{1 n}\right) \\
\ldots & \ldots & \ldots & \ldots & \ldots \\
\left(\underline{a}_{i 1}, \bar{a}_{i 1}\right) & \ldots & \left(\underline{a}_{i j}, \bar{a}_{1 j}\right) & \ldots & \left(\underline{a}_{i n}, \bar{a}_{i n}\right) \\
\ldots & \ldots & \ldots & \ldots & \ldots \\
\left(\underline{a}_{m 1}, \bar{a}_{m 1}\right) & \ldots & \left(\underline{a}_{m j}, \bar{a}_{m j}\right) & \ldots & \left(\underline{a}_{m n}, \bar{a}_{m n}\right)
\end{array}\right]
$$

and each normalize attributes can be shown as:

$$
\widehat{a}_{i j}=\frac{a_{i j}}{\underline{a}_{j}}
$$

for monotically increasing attributes and

$$
\widehat{a}_{i j}=\frac{\bar{a}_{j}}{a_{i j}}
$$

for monotonically decreasing attributes, where $\underline{a}_{j}$ and $\bar{a}_{j}$ are lowest possible and highest possible value for the $j^{t h}$ attribute respectively. The resulting normalized decision matrix $\widehat{D M}$ is shown below:

$$
\widehat{D M}=\left[\begin{array}{cccc}
\left(\hat{a}_{11}\right)^{w_{1}} & \left(\hat{a}_{12}\right)^{w_{2}} & \ldots & \left(\hat{a}_{1 n}\right)^{w_{n}} \\
\left(\hat{a}_{21}\right)^{w_{1}} & \left(\hat{a}_{22}\right)^{w_{2}} & \ldots & \left(\hat{a}_{2 n}\right)^{w_{n}} \\
\ldots & \ldots & \ldots & \ldots \\
\left(\hat{a}_{m 1}\right)^{w_{1}} & \left(\hat{a}_{m 2}\right)^{w_{2}} & \ldots & \left(\hat{a}_{m n}\right)^{w_{n}}
\end{array}\right]
$$

D. The Proposed Method of Network Selection Based on the Inferred Context of The User

Below is the summary of the proposed method:

1) Context aggregation.

2) Inferring the state of the user in terms of the predefined modes of operations (profile).

3) Determine the weight of each attribute in the inferred mode using the influence function.

4) Use the modified WPM to rank the alternatives.

5) The alternative with the greatest preference number is chosen. 


\begin{tabular}{l|ccccccc} 
Profile & BW & Delay & Power & $\begin{array}{c}\text { Packet } \\
\text { loss }\end{array}$ & Cost & Security & Jitter \\
\hline High BW & 0.77 & 0.08 & 0.04 & 0.05 & 0.02 & 0.04 & 0.01 \\
Low cost & 0.09 & 0.06 & 0.10 & 0.20 & 0.51 & 0.02 & 0.01 \\
Low power & 0.03 & 0.09 & 0.74 & 0.06 & 0.04 & 0.03 & 0.01 \\
Multimedia & 0.45 & 0.22 & 0.07 & 0.07 & 0.02 & 0.01 & 0.15 \\
Secure & 0.06 & 0.10 & 0.04 & 0.10 & 0.04 & 0.60 & 0.08 \\
& \multicolumn{7}{c}{ TABLE I }
\end{tabular}

WEIGHT DISTRIBUTION AMONG ATTRIBUTES FOR DIFFERENT USAGE PROFILES

\section{NumericAl EXAMPLES AND EVALUATION}

The purpose of this evaluation is first to validate our method against a well known decision making approach. The second objective is to demonstrate our method in a real use case example. To evaluate our method, in this section we have compared the WPM with respect to TOPSIS approach for typical usage profiles such as high bandwidth, low cost, and low power.

In this evaluation we have considered five access network alternatives. For simplicity of presentation, we have limited the alternatives to five network alternatives which characteristics are listed under $n$ number of attributes such as delay, bandwidth, cost, jitter, security, packet loss ratio and power constraints. The results show that ranking of candidate networks for TOPSIS and WPM are identical while WPM is a more robust method for a dynamic decision making situation and does not suffer from ranking abnormality.

One of the assumptions in this evaluation is that attributes belong to independent axis of measurements. This is to make the TOPSIS approach valid. The weight distribution is calculated based on the QoC for each attribute and the sensitivity analysis. For the purpose comparison, the same weights are assigned to attributes for the case of TOPSIS.

\begin{tabular}{l|ccccccc}
$\begin{array}{l}\text { Access } \\
\text { Networks }\end{array}$ & BW & Delay & Power & $\begin{array}{c}\text { Packet } \\
\text { loss }\end{array}$ & Cost & Security & Jitter \\
\hline Alt\# 1 & 0.4 & 1 & 0.9 & 0.3 & 0.2 & 0.4 & 0.3 \\
Alt\# 2 & 0.6 & 0.2 & 0.9 & 0.8 & 0.5 & 0.1 & 0.1 \\
Alt\# 3 & 0.3 & 0.9 & 0.2 & 0.5 & 0.1 & 0.7 & 0.8 \\
Alt\# 4 & 0.2 & 0.5 & 0.6 & 0.4 & 0.2 & 0.5 & 0.9 \\
Alt\# 5 & 0.5 & 0.2 & 0.5 & 0.6 & 0.1 & 0.8 & 0.7
\end{tabular}

TABLE II

CHARACTERISTICS OF EACH ACCESS NETWORK ALTERNATIVE. ATTRIBUTE VALUES OF ALTERNATIVES SCALED ON $[0,1]$ ON THE LEVEL OF DESIRABILITY

\begin{tabular}{l|ccccc}
$\begin{array}{l}\text { Access } \\
\text { Networks }\end{array}$ & $\begin{array}{c}\text { High BW } \\
\text { Profile }\end{array}$ & $\begin{array}{c}\text { Low Cost } \\
\text { Profile }\end{array}$ & $\begin{array}{c}\text { Low Power } \\
\text { Profile }\end{array}$ & $\begin{array}{c}\text { Multimedia } \\
\text { Streaming }\end{array}$ & $\begin{array}{c}\text { Secure } \\
\text { Profile }\end{array}$ \\
\hline Alt\# 1 & 0.43 & 0.30 & 0.75 & 0.48 & 0.42 \\
Alt\# 2 & 0.52 & 0.53 & 0.68 & 0.37 & 0.17 \\
Alt\# 3 & 0.33 & 0.20 & 0.25 & 0.44 & 0.60 \\
Alt\# 4 & 0.24 & 0.28 & 0.53 & 0.35 & 0.47 \\
Alt\# 5 & 0.46 & 0.22 & 0.44 & 0.42 & 0.60
\end{tabular}

TABLE III

PREFERENCE VALUE FOR RANKING OF EACH ALTERNATIVE FOR DIFFERENT PROFILES
The weight assignment can be done according to the method described in Section IV-B. Figure 2 shows the weight assignment with respect to the accuracy of the collected information based on the QoC parameters. The $\mathrm{x}$-axis is the variation of $\delta$ that indicates the sensitivity of a decision criteria. It denotes the threshold value of the ratio of change (can also be in \%) in the $k^{t h}$ attribute that can enforce a change in ranking of the access network alternatives. Lower $\delta$ refers to more important criterion that makes the decision more sensitive to changes of that criterion.

Assuming that the status of the user is inferred based on the aggregated context from various sources and assigning weights to each attribute based on the QoC and containment of that attribute (criterion) in inferred status or profile. Table I shows the weight distribution for our simulation.For each profile the features that have the highest impact are weighted more. Table $\mathrm{V}$ shows the five network alternatives under consideration and their features and attributes. For the purpose of the WPM, we eliminated the units by mapping each attribute to the scale range of $(0,1]$ where a scale of 1 represents superior choice. Ranking of alternatives are shown in table III in terms of preference value of each alternative for each usage profile.

Figure 1 shows that comparing the TOPSIS and WPM, the decision results of both agree with varying preference values.

\section{SUMmARY AND CONCLUSION}

Context-aware computing in mobile environment is interesting in that it paves the way for services and applications to take advantage of user contextual information such as time, location, activities and etc. One of the key aspects of an efficient context management framework is making use of the quality of contextual information. The QoC parameters are used for the weight distribution method that can affect the influence of an attribute on the final decision. We have shown our proposed SOA based system architecture for a context management framework. In this paper we have addressed the problem of dynamic decision for access network selection in heterogeneous network environments. Our proposed method is based on WPM decision making and weight assignment procedure is primarily based on QoC parameters and is also based on sensitivity analysis for the most influential criteria based on the state of user at a given time. The WPM for MADM is a more robust approach for dynamic decision making and it penalizes the attributes with poor quality to a greater extent. Furthermore, we take into account the QoC parameters and fuzzy saliency measure of the context information to facilitate 

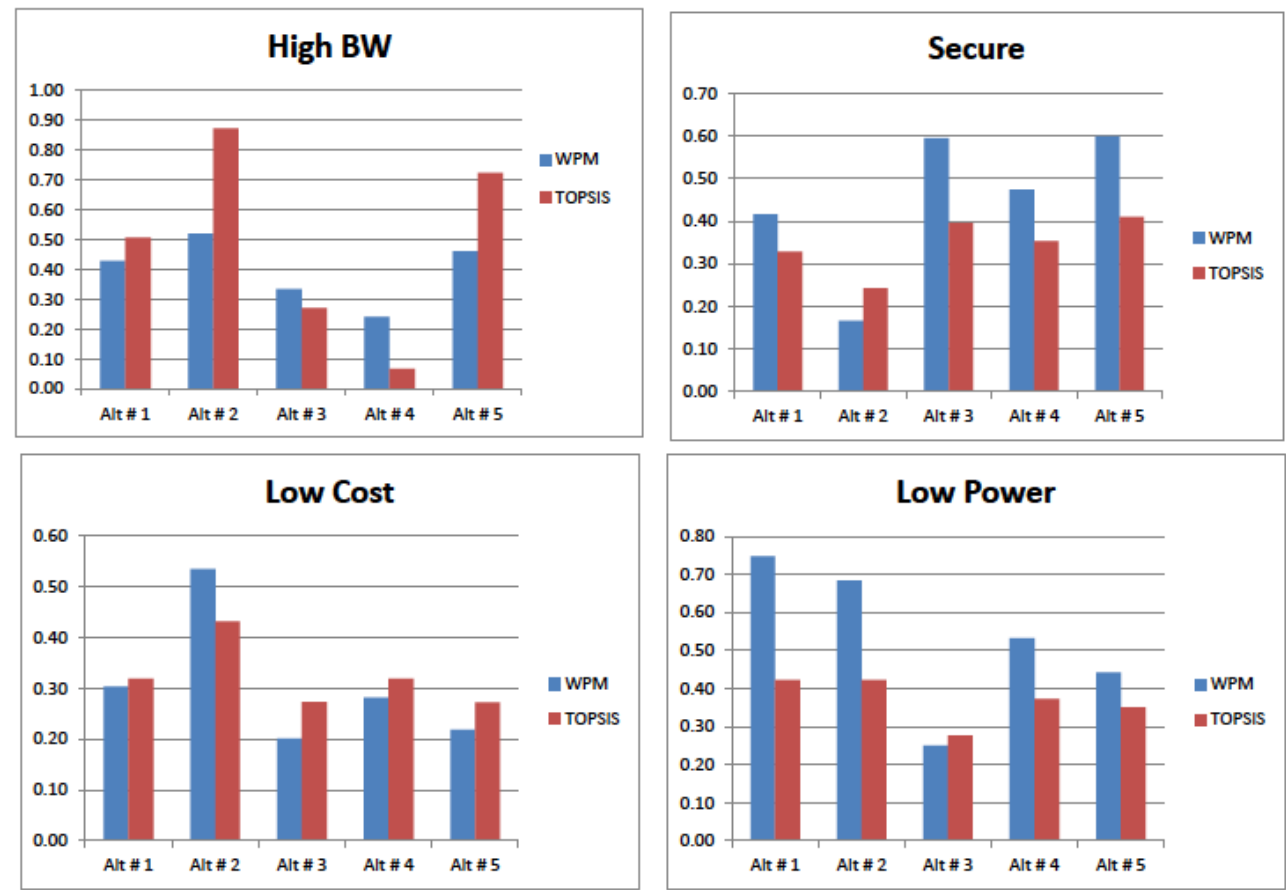

Fig. 1. Result of the network selection considering each usage profile

the distribution of weights for attributes in the MADM. QoC parameters are important since they can play a major role in determining the truth value of a context information. The evaluation and use case example of our method are shown in Section $\mathrm{V}$ and the weight assignment process and performance of WPM and TOPSIS are examined.

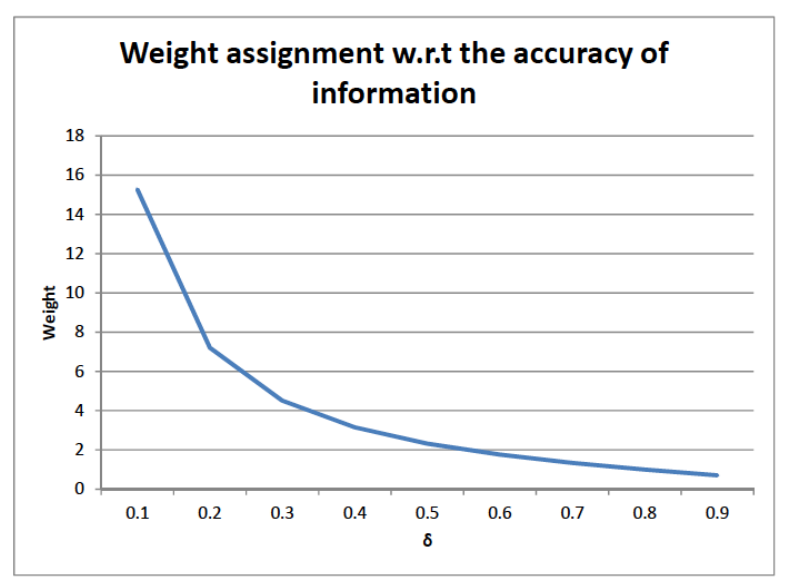

Fig. 2. weight assignment with respect to the accuracy of the collected information based on the $\mathrm{QoC}$ parameters

\section{ACKNOWLEDGMENT}

This work was supported in part by TELUS and the Institute for Computing, Information and Cognitive Systems (ICICS) at UBC.

\section{REFERENCES}

[1] M. Chen, S. Gonzalez, V. Leung, Q. Zhang, and M. Li, "A 2G-RFIDbased e-healthcare system," Wireless Communications, IEEE, vol. 17, no. 1 , pp. 37-43, 2010.

[2] J. Manner, M. Kojo, T. Suihko, P. Eardley, and D. Wisely, "Mobility related terminology," 2004.

[3] E. Triantaphyllou and C. Lin, "Development and evaluation of five fuzzy multiattribute decision-making methods," International Journal of Approximate Reasoning, vol. 14, no. 4, pp. 281-310, 1996.

[4] T. Erl, "SOA: Principles of service design," 2007.

[5] A. Padovitz, S. Loke, and A. Zaslavsky, "Towards a theory of context spaces," in Pervasive Computing and Communications Workshops, 2004. Proceedings of the Second IEEE Annual Conference on. IEEE, 2004, pp. $38-42$

[6] A. Padovitz, S. Loke, A. Zaslavsky, B. Burg, and C. Bartolini, "An approach to data fusion for context awareness," Modeling and Using Context, pp. 353-367, 2005.

[7] B. Schilit, "A context-aware system architecture for mobile distributed computing," Unpublished PhD, Columbia University, 1995.

[8] H. Sugano, S. Fujimoto, G. Klyne, A. Bateman, W. Carr, and J. Peterson, "Presence information data format (PIDF)," draft-ietf-impp-cpim-pidf-08 (work in progress), 2003.

[9] J. Rosenberg, H. Schulzrinne, G. Camarillo, A. Johnston, J. Peterson, R. Sparks, M. Handley, and E. Schooler, "RFC3261: SIP: session initiation protocol," RFC Editor United States, 2002.

[10] D. Box, D. Ehnebuske, G. Kakivaya, A. Layman, N. Mendelsohn, H. Nielsen, S. Thatte, and D. Winer, "Simple object access protocol (SOAP) 1.1," 2000

[11] Y. Kim and K. Lee, "A quality measurement method of context information in ubiquitous environments," in Hybrid Information Technology, 2006. ICHIT'06. International Conference on, vol. 2. IEEE, 2006, pp. $576-581$.

[12] A. Manzoor, H. Truong, and S. Dustdar, "On the evaluation of quality of context," Smart Sensing and Context, pp. 140-153, 2008.

[13] T. Buchholz, A. Kupper, and M. Schiffers, "Quality of context: What it is and why we need it," in Proceedings of the Workshop of the HP OpenView University Association. Citeseer, 2003.

[14] S. Chen, C. Hwang, M. Beckmann, and W. Krelle, Fuzzy multiple attribute decision making: methods and applications. Springer-Verlag New York, Inc. Secaucus, NJ, USA, 1992. 